\title{
Effect of Chitosan Loading on the Morphological, Thermal, and Mechanical Properties of Diglycidyl Ether of Bisphenol A/Hexamethylenediamine Epoxy System
}

\author{
B. Satheesh, ${ }^{1}$ K. Y. Tshai, ${ }^{2}$ and N. A. Warrior ${ }^{1}$ \\ ${ }^{1}$ University of Nottingham, University Park, Nottingham NG7 2RD, UK \\ ${ }^{2}$ University of Nottingham Malaysia Campus, Jalan Broga, 43500 Semenyih, Selangor, Malaysia \\ Correspondence should be addressed to K. Y. Tshai; kim-yeow.tshai@nottingham.edu.my
}

Received 19 June 2014; Revised 10 September 2014; Accepted 24 September 2014; Published 30 October 2014

Academic Editor: Suying Wei

Copyright (c) 2014 B. Satheesh et al. This is an open access article distributed under the Creative Commons Attribution License, which permits unrestricted use, distribution, and reproduction in any medium, provided the original work is properly cited.

\begin{abstract}
The effect of chitosan filled diglycidyl ether of bisphenol A (DGEBA) epoxy system were investigated using the thermal, mechanical, and morphological properties. The mixing ratio of resin/hardener was kept constant while the chitosan of 1.0, 2.5, 5.0, 7.5, and 10 weight percentage (wt\%) was incorporated into the system. The thermal stability and the transition behaviour of the chitosan filled epoxy system were analysed through a differential scanning calorimetry (DSC), thermogravimetric analysis (TGA), and Fourier transform infrared spectroscopy (FTIR) while atomic force microscope (AFM) and scanning electron microscopy (SEM) were used to investigate the morphology. It was observed that the additive tends to agglomerate, with the formation of clear phase separation, when the chitosan content increases above $5 \mathrm{wt} \%$. At lower chitosan loading ( $2.5 \mathrm{wt} \%$ and below), relatively uniform dispersion of the additive can be achieved. The thermal stability of the system increases with chitosan loading while the mechanical tensile strength is compromised.
\end{abstract}

\section{Introduction}

Epoxy resins are molecules containing one or more $\alpha$ - or 1 , 2-epoxide groups, which can be crosslinked to form threedimensional (3D) thermoset network structure. The degree of crosslinking and hence the material properties can be controlled by varying the amount of the resin hardener system, controlled curing over a wide range of temperatures and introduction of additives or fillers. Epoxy has been extensively used in a wide range of industrial applications owing to its excellent adhesiveness, resistance to heat and common chemical, mechanical strength, and electrical insulating properties. However, the material remains excessively brittle once cured and suffers from low impact strength, poor fracture toughness, and resistance to crack propagation. Dušek et al. [1] pointed out that alternating mechanism of network formation in DGEBA amine hardener system does not offer the potential of partial segregation for the formation of inhomogeneous crosslinking. On the other hand, more pronounced inhomogeneity may be promoted through thermodynamic incompatibility or nonalternating curing mechanisms in more complicated thermosetting systems. Over the past decade, various attempts to enhance the toughness of epoxy system have been carried out by incorporating fillers into epoxidesto produce a variant form of epoxy resin coupled with different chemical compositions. Review of the literature shows that majority of the previous work employed thermoplastic, rubber, and elastomer compounds as toughening agents in epoxy systems [2-13].

Zhang and $\mathrm{Yu}$ [5] observed that addition of semicrystalline isotactic poly(phenyl glycidyl ether) (i-PPGE) modifier resulted in change of particle size distribution, critical intensity factor, and crystallinity. Ochi et al. [6] recorded a measureable level of improvement in toughness by introducing a reactive elastomer carboxyl terminated liquid butadiene-acrylonitrile rubber (CTBN). Nigam et al. [7] used a carboxyl terminated polybutadiene (CTPB) on novolac epoxy resin which demonstrated enhancement in the material toughness in tensility, flexure, and impact. Pereira Soares et al. [8] used hydroxyl-terminated polybutadiene (HTPB) 
TAble 1: Properties of the Hexamethylenediamine (HMDA) hardener.

\begin{tabular}{lcccccc}
\hline $\begin{array}{l}\text { Molecular Weight } \\
{[\mathrm{g} / \mathrm{mol}]}\end{array}$ & $\begin{array}{c}\text { Melting Point. } \\
{\left[{ }^{\circ} \mathrm{C}\right]}\end{array}$ & $\begin{array}{c}\text { Boiling Point. } \\
{\left[{ }^{\circ} \mathrm{C}\right]}\end{array}$ & $\begin{array}{c}\text { Flash Point } \\
{\left[{ }^{\circ} \mathrm{C}\right]}\end{array}$ & $\begin{array}{c}\text { Vapour Pressure } \\
{[\mathrm{kPa}]}\end{array}$ & $\begin{array}{c}\text { Water Solubility } \\
{[\mathrm{g} / \mathrm{L}]}\end{array}$ & $\begin{array}{c}\text { Additives } \\
\text { Impurities }\end{array}$ \\
\hline 116.24 & 41 & 250 & 80 & $\begin{array}{c}0.05[0.4 \mathrm{mmHg}] \\
\text { at } 25^{\circ} \mathrm{C} \mathrm{CAL}\end{array}$ & 800 at $15.6^{\circ} \mathrm{C}$ & None \\
\hline
\end{tabular}

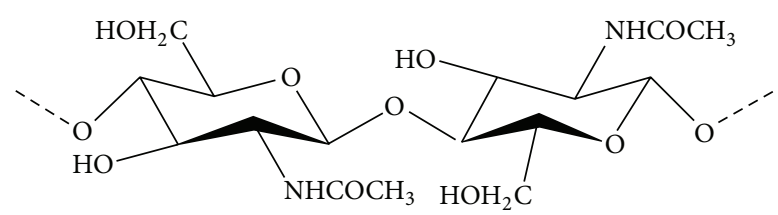

FIGURE 1: Chitin molecular structure [14].

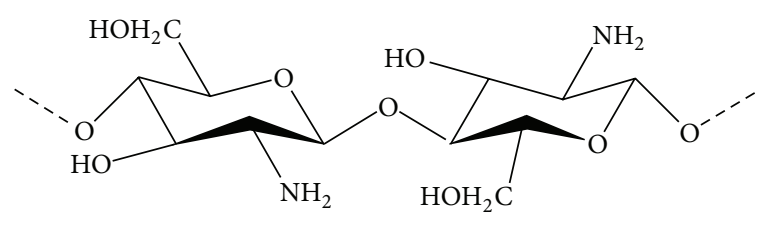

FIgURE 2: Deacetylated chitosan molecular structure [14]. elastomer as toughening agent which showed that it is possible to obtain a $100 \%$ increase in impact resistance with HTPB concentration up to $5 \mathrm{phr}$ (parts per hundred rubber). Liu et al. [9] studied the effects of curing agents, that is, 4,4diaminodiphenyl methane (DDM), dicyandiamide (DICY), and diethylphosphite (DEP), on the 4-(N-maleimidophenyl) glycidyl ether (MPGE) which revealed that the crosslinked material possesses high glass transition, good thermal stability, and attractive flame retardant properties. Zhang [10] explained the advantages and disadvantages of using reactive liquid rubbers, functionally terminated engineering thermoplastics, reactive ductile diluents, and inorganic and hybrid particles as modifiers to improve fracture toughness. Cai et al. [11] conducted experiments on DGEBA epoxy resin with isocyanate terminated polyethers (ITPE) modifier showing that the thermal stability decreases with the introduction of ITPE while the impact and flexural strength of the cured modified epoxy increase with ITPE loading. Brooker et al. [12] incorporated thermoplastic toughener poly(ether sulfone) copolymer into epoxy system reporting that the ultimate tensile strength and fracture toughness increased with increasing thermoplastic content. Pearson and Yee [13] modified DGEBA with thermoplastic poly(phenylene oxide) (PPO) finding that the fracture toughness improved with PPO content in a linear fashion.

One of the most abundant and easily accessible naturally occurring polysaccharides commonly found in the exoskeleton of shrimps and crabs, chitin (2-Acetamido-2deoxy-D-glucose, Figure 1), is a linear high molecular weight crystalline polysaccharide and, like cellulose, is made of $\beta$ $(1 \rightarrow 4)$ linked D-glucose. Chitosan (a biomedical cationic amino polysaccharide filler) is the $\mathrm{N}$-deacetylated derivative of chitin [14] which, instead of the acetyl group in chitin, has the $-\mathrm{NH}_{2}$ chain (Figure 2). The presence of high nitrogen content $(6.89 \%)$ in chitosan makes it feasible for use with epoxies while its antimicrobial properties provide added advantages. In the present study, chitosan is chosen as a modifier to DGEBA epoxy resin with hexamethylenediamine (HMDA) as a hardener to investigate the effects of chitosan loadings on the morphological, thermal, and mechanical properties of the resulting cured system.

\section{Materials}

2.1. Epoxy Resin. A room temperature curable DGEBA resin with low viscosity, EPOFINE 564 (viscosity ranging between 400 and $600 \mathrm{MPa} \cdot \mathrm{s}$ at $25^{\circ} \mathrm{C}$ ), suitable for the manufacture of composite tools and lamination was supplied by Fine Finish Organics Pvt. Ltd., India.

2.2. Coreactants/Hardener. Hexamethylenediamine (HMDA) was obtained from Planatec Lab Solutions, Hyderabad, India. The material was produced by hydrogenation of adiponitrile and the properties of HMDA are given in Table 1.

2.3. Chitosan. The chitosan used in this work was supplied by India Sea Foods, Kochi, India. The process of extracting chitosan was carried out industrially by the supplier. Typical production steps involved are demineralisation, deproteinisation, decolouration, and deacetylation. The deacetylation percentage depends on chitosan extraction method and requires careful chemical treatment. The chitosan used in this research was extracted from shrimps and the average chitin content in shrimp shells was $17 \%$. The shells extracted from shrimps were first washed and then ground to powder with a size particle of $0.3-0.5 \mathrm{~mm}$. The powdered form of shrimp shells contains high percentages of chitin, calcium carbonate $\left(\mathrm{CaCO}_{3}\right)$, and proteins. The first stage of chemical process starts with demineralisation where $\mathrm{CaCO}_{3}$ is removed using $7 \%$ hydrochloric acid $(\mathrm{HCl})$, in the ratio of $1 \mathrm{~g}$ of powdered shell to $10 \mathrm{~mL}$ of $\mathrm{HCl}$, and the mixture is left for $24 \mathrm{hrs}$ in room temperature. Upon demineralisation, deproteinisation takes place where the demineralised powder is subjected to $10 \%$ sodium hydroxide $(\mathrm{NaOH})$ treatment, in the ratio of $1 \mathrm{~g}$ of demineralised powder to $10 \mathrm{~mL}$ of $\mathrm{NaOH}$, and the mixture is treated at $60^{\circ} \mathrm{C}$ for $24 \mathrm{hrs}$ to yield chitin. Deacetylation of chitin is carried out to remove the acetyl group, where the extracted chitin is treated with $50 \% \mathrm{NaOH}$ at $60^{\circ} \mathrm{C}$ for $8 \mathrm{hrs}$. The deacetylation achieved around $73 \%$ of chitosan in powder form. The chitosan powder is sun-dried and the final product can now be used as filler in the epoxy-hardener system. 
TABLE 2: Weight percentage (wt\%) of chitosan.

\begin{tabular}{lcccccc}
\hline Sample Group & A & B & C & D & E & F \\
\hline Chitosan [wt\%] & 0.0 & 1.0 & 2.5 & 5.0 & 7.5 & 10.0 \\
\hline
\end{tabular}

\section{Methodology}

The preparation of mixture containing epoxy-hardener system with chitosan was performed at ambient temperature. Curing is an exothermic process and the cure time is dependent on three parameters: (a) the weight of the epoxy resin used, (b) the weight of the hardener used, and (c) the cure temperatures and the surrounding environment.

Epoxy and hardener weights are kept constant at $31 \mathrm{~g}$ and $9 \mathrm{~g}$, respectively, whereas the wt $\%$ of chitosan was varied in the fabrication, as shown in Table 2.

The specified quantity of epoxy resin is first mixed with chitosan powder using a magnetic stirrer maintained at $300 \mathrm{rpm}$ for 15 mins, after which the predetermined amount of HMDA is added to the mixture and further stirred for another 10 mins. The resulting mixture was subsequently used for DSC analysis and to produce cured specimens for mechanical test, TGA, SEM, FTIR, and AFM in accordance with the procedures described below.

For DSC analysis, the resulting mixture in the range of 7-10 $\mathrm{mg}$ was held within a dedicated sample pan. The sample was scanned between room temperature and up to $350^{\circ} \mathrm{C}$, at a heating rate of $10^{\circ} \mathrm{C} / \mathrm{min}$, and the heat flux versus temperature data was recorded.

For the fabrication of cured specimens, the resulting mixture was poured onto glass mould of dimensions $15 \mathrm{~cm} \times$ $15 \mathrm{~cm} \times 3 \mathrm{~cm}$ where the mould surface was precoated with silicon oil. Curing was allowed to take place within the glass mould to yield cured specimens of varying chitosan loadings.

TGA was carried out on a $10.0 \pm 5.0 \mathrm{mg}$ specimen to investigate the thermal degradation behaviour of the samples and to observe the maximum mass loss. The heating temperature was set within the range $25-850^{\circ} \mathrm{C}$, ramped at a rate of $20^{\circ} \mathrm{C} / \mathrm{min}$ and under nitrogen flow rate at $50 \mathrm{~mL} / \mathrm{min}$.

Specimens for SEM micrographs were prepared by cryogenically fracturing the composite in liquid nitrogen. The samples were then sputter-coated with gold and micrograph images were taken under low vacuum mode, $10 \mathrm{kV}$ voltages and 220x magnification power within a Hitachi S-2400 SEM device.

For the AFM imaging, a flat fragment of the specimen was securely held onto the centre of the steel sample puck using a 2-sided adhesive tape and subsequently mounted on the scanner tube. Contact mode AFM topographical scans using silicon nitride cantilevers probe of $500 \mathrm{~nm}$ in radius were conducted on the composite specimen.

FTIR analysis was performed using a Thermo Nicolet Avatar370 spectrometer to identify the resulting chemical bonds of chitosan filled epoxy system. The cured specimen was ground into powder using mortar and pestle. The finely grinded specimen powder was subsequently mixed with potassium bromide $(\mathrm{KBr})$ powder in the ratio of $1: 50$ and

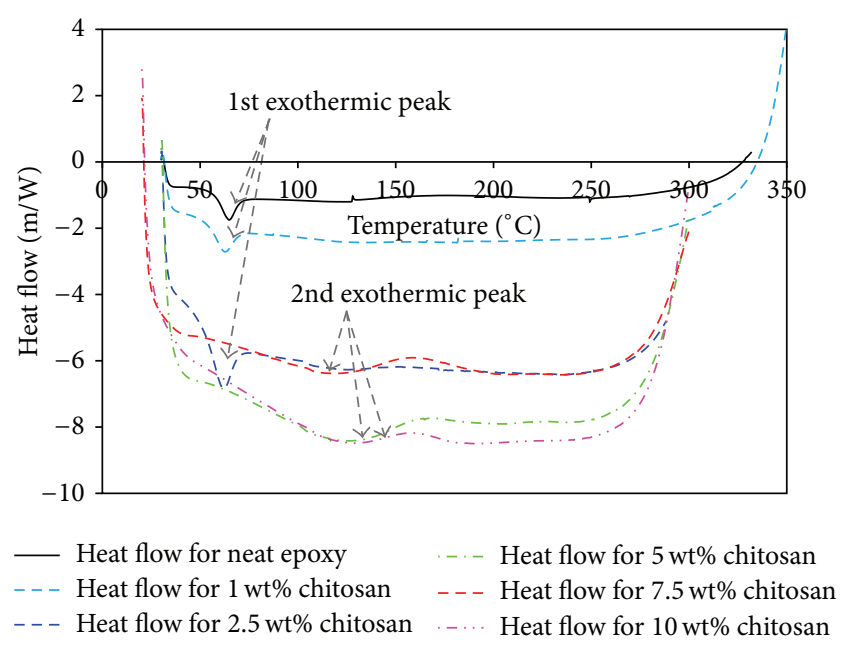

FIgURE 3: DSC results of epoxy/hardener sample with varying chitosan loadings.

pressed to a thin film pellet using a dye and bolt press assembly. Each active group of the component in the pellet has its own characteristic absorbance or transmittance peak to a specific infrared (IR) spectrum and the concentration of each component can be calculated from the area under its peak. $\mathrm{KBr}$ pellets with varying chitosan loadings were subjected to FTIR scan at IR spectrum from 800 to $4000 \mathrm{~cm}^{-1}$.

The tensile properties were determined using a Shimadzu AG-1 universal testing machine equipped with a $50 \mathrm{kN}$ load cell. Dumbbell specimens corresponding to BSI BS 903-A2 were prepared using a standard cutting die. The measurements were carried out at a crosshead speed of $50 \mathrm{~mm} / \mathrm{min}$ according to ASTM D638 standard.

\section{Results and Discussion}

4.1. DSC Analysis. A plot of the DSC heat flow versus temperature results is shown in Figure 3. It can be observed that the heat enthalpy curves with chitosan loading lower than $2.5 \mathrm{wt} \%$ revealed distinctive single exothermic peak at temperature around $60^{\circ} \mathrm{C}$ and the peak area increases with chitosan loading. For composites with chitosan loading greater than $2.5 \mathrm{wt} \%$, the exothermic peak area around $60^{\circ} \mathrm{C}$ is much reduced and an additional second exothermic peak appears at around $125^{\circ} \mathrm{C}$. This reaction is predominantly induced by the presence of excessive primary amines groups along the chitosan macromolecules that react with the epoxy groups in excess until maximum conversion. The second peak at higher chitosan loading is identified as the chitosan phase induced chemical cure of the sample.

As chitosan is not soluble in epoxy system, the incorporation of chitosan filler tends to induce phase separation in epoxy. However, at low concentration the agglomeration of chitosan is relatively less noticeable. It is only at higher loadings that the agglomeration phenomenon is exposed with the presence of a second peak. Similar observations were reported by numerous researchers $[4,15,16]$, where 


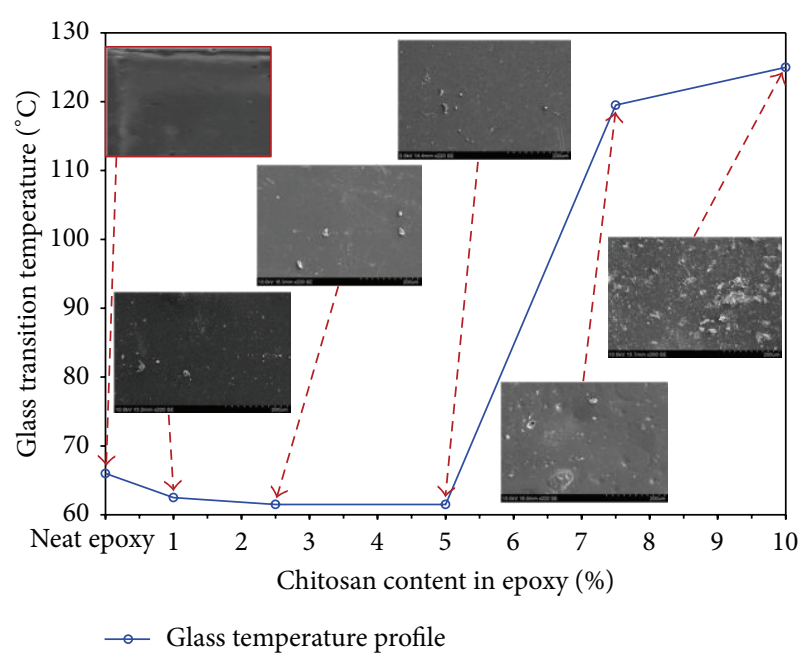

FIGURE 4: Variation of glass transition temperature with varying chitosan content. SEM images demonstrate that the extent of agglomeration tends to increase with increased chitosan content.

chemical reaction involved a more dominant additive in epoxy systems causing the existence of a second phase. The utilisation of HMDA hardener and the introduction of excessive free amine groups through chitosan chains may have improved the adhesion between constituent components. However agglomeration begins to form in samples with higher concentrations of chitosan.

The existence of a second phase, that is, chitosan phase, is more evident in the plot of variation in glass transition temperature, $T_{g}$, in Figure 4. It is demonstrated that the changes in $T_{g}$ for specimens with 1.0, 2.5, and $5.0 \mathrm{wt} \%$ of chitosan are negligible. However, a steep increase in $T_{g}$ can be seen in specimens with chitosan loading higher than $5.0 \mathrm{wt} \%$, recording $119.2^{\circ} \mathrm{C}$ and $124.4^{\circ} \mathrm{C}$ for $7.5 \mathrm{wt} \%$ and $10 \mathrm{wt} \%$ of chitosan, respectively. The increase in $T_{g}$ confirmed that the epoxy composite reaches to the maximum conversion with excessive presence of chitosan.

SEM images taken at each of the respective chitosan loadings demonstrate that nonhomogeneous mixture was obtained when low concentration of chitosan filler is mixed with the epoxy system. At higher loadings, it is evident that the chitosan phase is more dominant with rougher surface due to increased agglomeration.

The recorded improvement on thermal stability may be related to the collective effect of reduced intermolecular space and bulky filler as a result of the agglomeration at higher chitosan loading. In the work of Shokralla and Al-Muaikel [17], the authors conducted an investigation on the effects of fillers in varying thermal properties of epoxy/phenolic blends. It was observed that free volume is much reduced at higher filler loadings, incurring additional restriction to molecular movement and rotation, which in turn causes the loss of homogenous mixture and agglomeration takes place. This phenomenon of space restriction was found capable of improving thermal stability through enhanced intermolecular vibrations. Similar observation was recorded

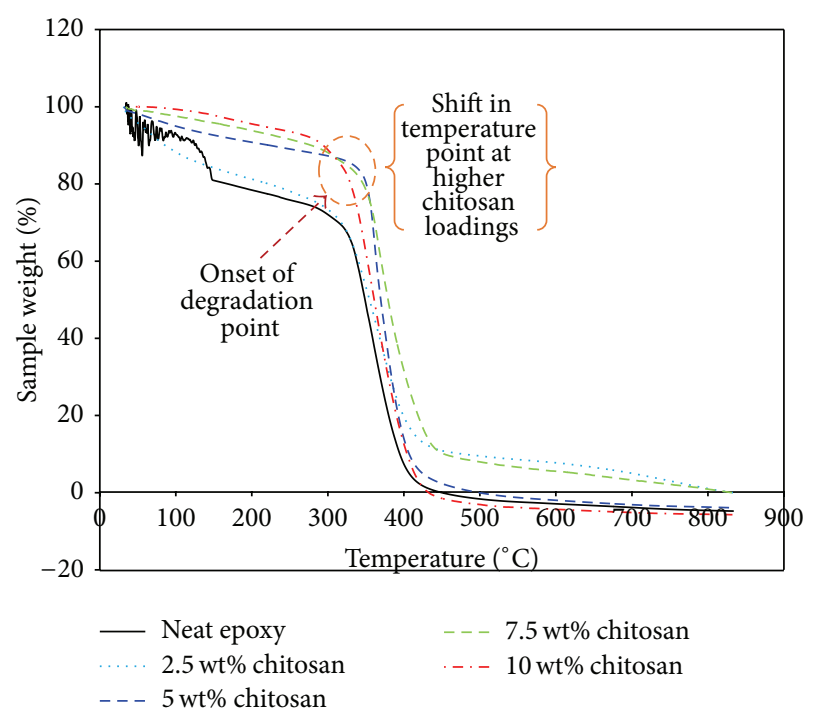

FIGURE 5: TGA curve of specimen with varying wt\% of chitosan in DGEBA/hardener system.

by Park et al. [18] where the authors demonstrated that larger fillers were capable of improving thermal stability by restricting molecular movement and rotation. It is hence evident that the chitosan phase is more dominant in specimens with higher filler content, causing the agglomeration of bulky particles. This phenomenon highlighted a great space reduction within DGEBA/HMDA and thus improved thermal stability. From the SEM images, it can be observed that the space within the chitosan filled epoxy system is clearly reduced thereby restricting molecular movement.

4.2. Thermogravimetric Analysis (TGA). It can be seen from Figure 5 that the thermal stability of chitosan filled epoxy composite (found from the onset of degradation point) increases with chitosan loading. An increase of approximately $10^{\circ} \mathrm{C}$ is observed in the specimen with 5.0 and $7.5 \mathrm{wt} \%$ of chitosan. The increase in thermal stability may be associated with the increase in crosslinking of the epoxy with chitosan. As chitosan is a cycloaliphatic amine; the amine groups present in chitosan could participate in the crosslinking reactions with epoxy, hence imparting a higher thermal stability. However, in the case of $10 \mathrm{wt} \%$ chitosan loading, the agglomeration of the bulky chitosan particles reduces its probability to participate in the crosslinking reaction. Thereby, a reduced efficiency in enhancing thermal stability of the composite is seen as the onset of degradation point which is slightly below that of the $7.5 \mathrm{wt} \%$ chitosan. The results show that an increase in the chitosan content does improve thermal stability but at excessive loadings, that is, at $10 \mathrm{wt} \%$ and above, the shift in temperature shows that agglomeration leads to chitosan phase dominating the reaction, causing poor crosslinking and a drop in thermal stability. At higher loadings, weight of the molecules increases and this was due to the agglomeration as exhibited by the SEM images. Increase in molecular weight could also have played a role in improving thermal stability. 


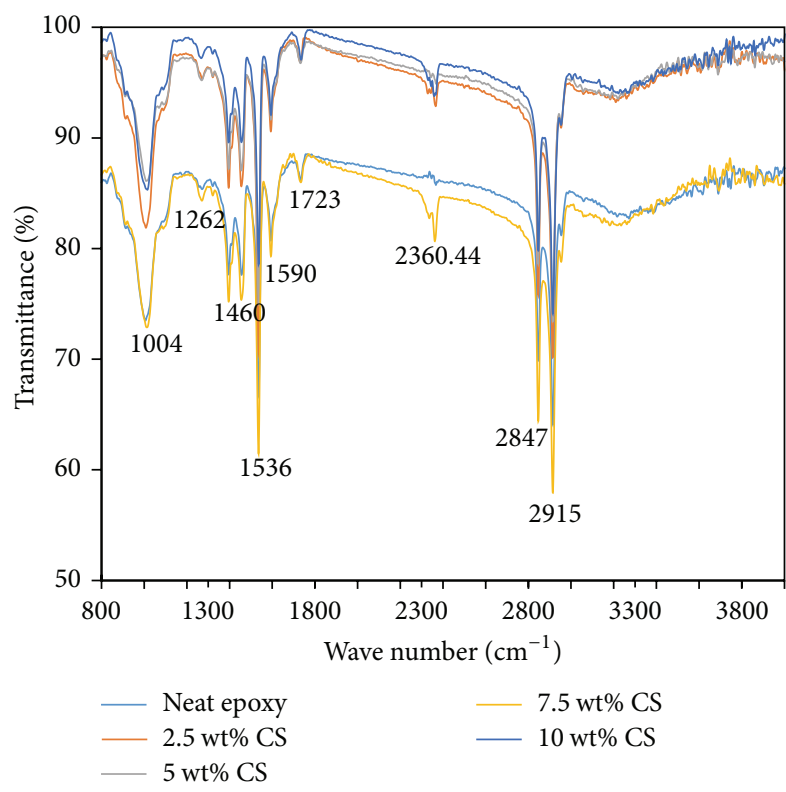

FIGURE 6: FTIR spectra showing material interaction at different wt $\%$ of chitosan loading.

4.3. Fourier Transform Infrared Spectroscopy (FTIR). The FTIR absorption spectra are displayed in Figure 6. Significant absorbance was observed at 2915, 2847, 2360, 1723, 1590, 1536, $1460,1395,1262$, and $1004 \mathrm{~cm}^{-1}$. The peaks at $1004 \mathrm{~cm}^{-1}$ were assigned to the vibrational modes associated with saccharide units in chitosan while the peak at $1262 \mathrm{~cm}^{-1}$ was due to the chitosan hydroxyl $(-\mathrm{OH})$ group, which clearly indicates that the interaction of $-\mathrm{OH}$ groups has taken place $[19,20]$. Bands at 1723,1590 , and $1536 \mathrm{~cm}^{-1}$ show the amino group reactions from chitosan. Although chitosan contains a large portion of sugar molecules due to its polysaccharide nature, there are also amine groups present which make chitosan very reactive. The peaks at $1262-1004 \mathrm{~cm}^{-1}$ were a result of the $\mathrm{C}-\mathrm{O} / \mathrm{C}-\mathrm{N}$ stretching and the peak at $2360 \mathrm{~cm}^{-1}$ was assigned to nitrile groups stretching in the chitosan molecules $[19,20]$. It is apparent from the recorded spectra that the absorption peaks increase with chitosan loading. The higher FTIR absorption rate observed at higher chitosan loadings can be related to the excessive presence of amines, which makes the samples highly reactive because of the stretching due to $\mathrm{C}-\mathrm{O} / \mathrm{C}-\mathrm{N}$ bonds occurs during chemical curing. Therefore, the nitrile and amine bonds could be related to the presence of second peak in the DSC curve and the agglomeration at higher chitosan loadings. Overall, the FTIR investigation showed evidences of intermolecular bonding between epoxy and chitosan particles. Amine reaction from chitosan and $-\mathrm{OH}$ group reactions were the most important reactions revealed from the current FTIR readings.

4.4. Atomic Force Microscopy (AFM). Figure 7 shows the AFM images of the composite with varying chitosan loadings.
It can be seen that relatively uniform filler distribution was achieved with lower wt $\%$ of chitosan, where the surface topography was found to be more homogenized. Smooth surfaces were observed for neat epoxy up to $2.5 \mathrm{wt} \%$ of chitosan loading, after which agglomeration is clearly visible. At $5 \mathrm{wt} \%$ of chitosan, the particles begin to agglomerate and revealed clear phase separation. This may be attributed to the hydrophobic-hydrophilic interaction between the epoxy and the chitosan filler at large concentrations. AFM results indicated that the insolubility and agglomeration of chitosan in epoxy system is increasingly obvious at higher loadings. Radhakumary et al. [21] and Rutnakornpituk et al. [22] attempted to address the reaction mechanism of chitosan molecule through a discussion on the high hydrophilicity nature of chitosan. The authors' work revealed that $-\mathrm{NH}_{2}$ molecules present in chitosan are capable of acting as reaction site for epoxy, which at low filler loadings provides the homogenisation effect, and such reaction was found to be similar to epoxy/hardener reaction in conventional crosslinked system. However, at higher filler loadings the hydrophobic-hydrophilic interactions between the epoxy and filler exceeded the interaction of the $-\mathrm{NH}_{2}$ functionality of chitosan.

4.5. Young's Modulus and Ultimate Tensile Strength (UTS). The Young's modulus was computed as the steepest slope in the stress-strain response of the composite and the result is depicted in Figure 8. It can be seen that the measured elastic modulus increases with chitosan loading but the magnitude dropped slightly in specimen loaded with $10 \mathrm{wt} \%$ of chitosan. With increasing chitosan loadings, the reaction becomes more amine rich and dominates the curing process, leading to a more rigid and brittle composite with higher modulus. Neat epoxy without chitosan on the other hand shows a reaction where the ring opening by the - $\mathrm{OH}$ groups took place with epoxy monomers. In the case with excessive chitosan, that is, $10 \mathrm{wt} \%$, the agglomerated bulky chitosan particle of size approx. $40 \mu \mathrm{m}$ reduced the probability of its participation in crosslinking, indicating a potential threshold of chitosan loading for modulus enhancement.

Figure 9 shows the ultimate tensile stress response at varying $\mathrm{wt} \%$ of chitosan. The results demonstrated that increasing the chitosan content had an effect on reducing the fracture strength of the epoxy thermoset. Excessive chitosan resulted in an amine rich formulation and is capable of enhancing crosslinking and therefore increases the UTS. However, in the scenario where agglomeration took place, the bulky chitosan molecules begin to dominate and the reaction probability of excess epoxy with free amine in chitosan dropped, leading to poor crosslinking, and subsequently the UTS dropped as a result. Notably adding chitosan alone to DGEBA without hardener induces material brittleness and fracture and this was found to occur prior to yielding. On the other hand, the introduction of amine hardener in the presence of chitosan leads to the formation of 3D networks. This tends to induce more ductile behaviour; however the UTS remained low due to chitosan agglomeration in the sample. 


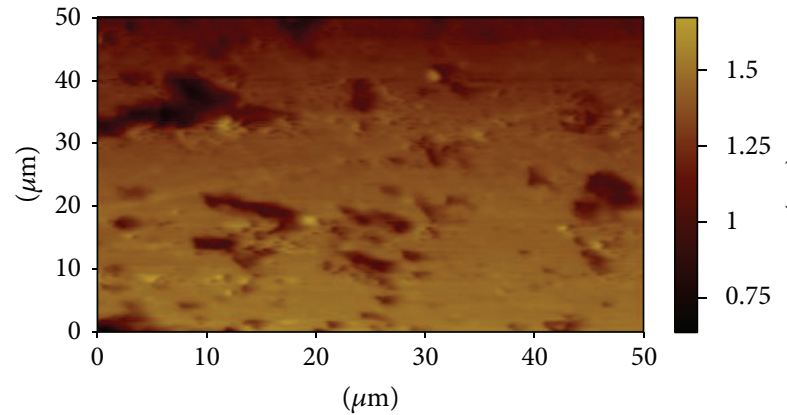

(a) Neat epoxy

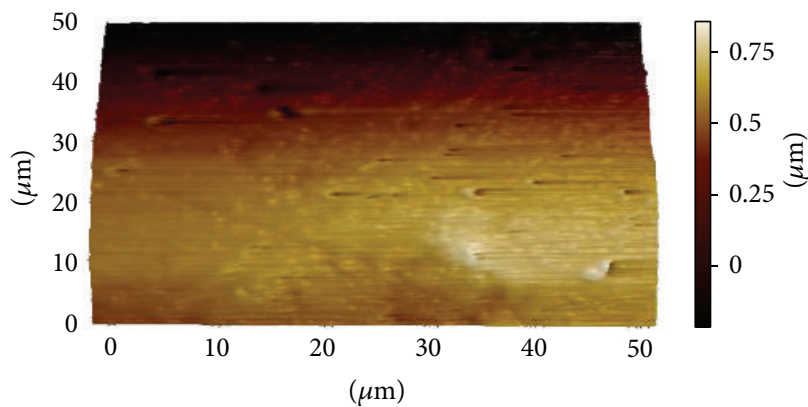

(c) $2.5 \mathrm{wt} \%$ chitosan

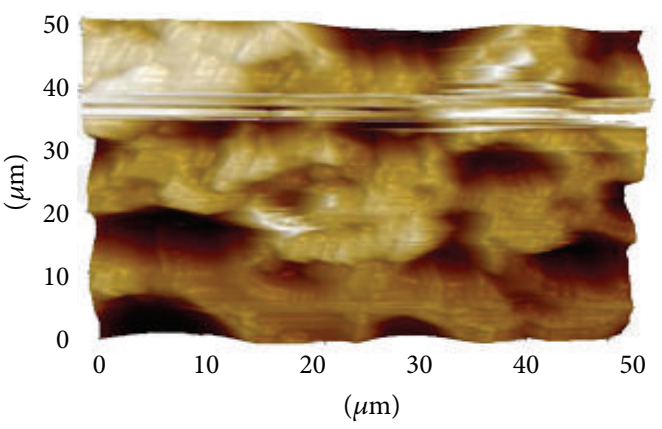

(e) $7.5 \mathrm{wt} \%$ chitosan

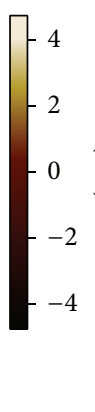

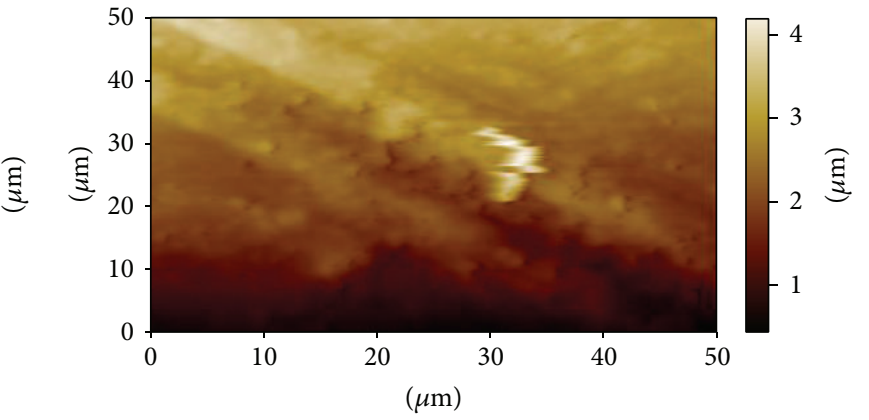

(b) $1 \mathrm{wt} \%$ chitosan

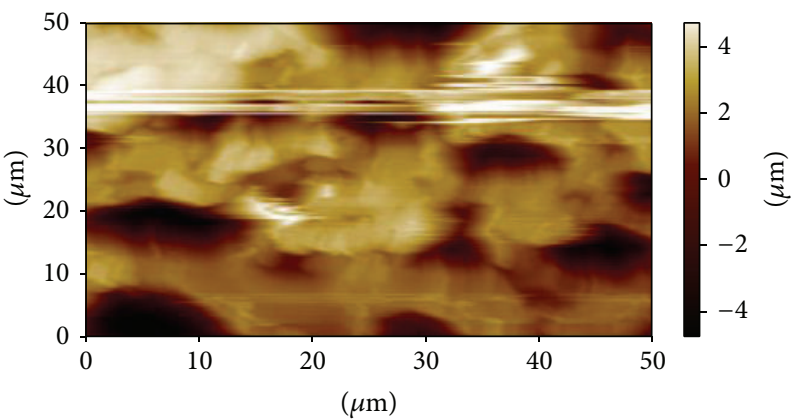

(d) $5 \mathrm{wt} \%$ chitosan

$$
\begin{array}{r}
\text { ह छ } \\
20 \\
10 \\
0 \\
0
\end{array}
$$$$
0 \quad 10
$$$$
10
$$

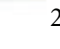

$(\mu \mathrm{m})$

(f) $10 \mathrm{wt} \%$ chitosan

FIGURE 7: AFM images comparing neat epoxy and epoxy with varying wt $\%$ of chitosan.

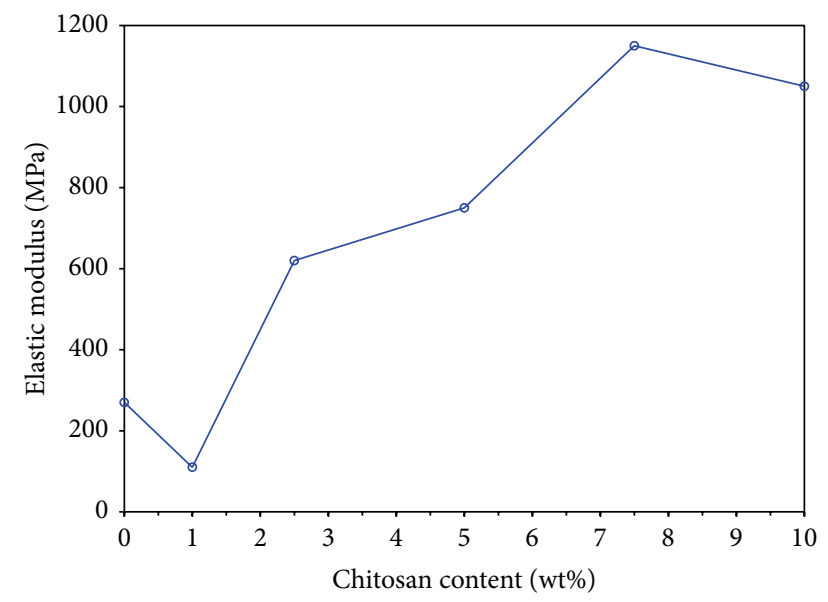

Figure 8: Young's modulus of epoxy specimen at varying wt $\%$ of chitosan.

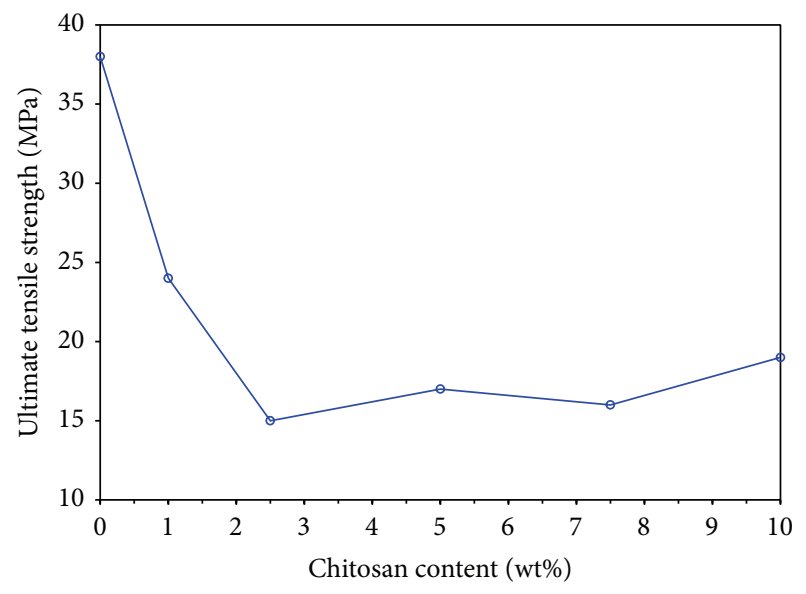

FIGURE 9: Ultimate tensile strength of epoxy specimen at varying wt $\%$ of chitosan. 


\section{Conclusions}

Chitosan obtained from the exoskeletons shells of shrimps is used as a filler to epoxy/hardener thermosetting polymer. The results successfully demonstrated the variation in thermal, morphological, and mechanical properties of the thermoset composite system with varying chitosan loadings ranging from 0 to $10 \mathrm{wt} \%$. The polycationic nature of chitosan with $-\mathrm{NH}_{2}$ and $-\mathrm{OH}$ groups was found capable of acting as the reaction site in producing a relatively homogenous mixture and reveals an improvement in thermal stability. However, the UTS are being compromised due to agglomeration at higher filler loadings and miscibility of chitosan molecules decreases. DSC scan indicates the presence of phase separation at higher chitosan loadings, manifesting as the second exothermic peak in the thermogram. The first exothermic peak denotes the homogenous phase whereas the second exothermic peak revealed the chitosan phase induced chemical cure of the epoxy system. SEM confirms that, by adding small quantity of chitosan to epoxy system, homogeneity of the mixture is disturbed while at higher chitosan loadings, clear phase separation is evident due to increase in agglomeration. AFM images further support the existence of this chitosan phase separation and agglomeration at higher loadings. The increase in $T_{g}$ at chitosan loadings greater than $5.0 \mathrm{wt} \%$ can be attributed to its hydrophilic nature and immiscibility with epoxy, whereby the bulky agglomerated molecular size of chitosan greatly restricted mobility during curing reaction and the enhanced intermolecular vibrations capable of improving thermal stability. The FTIR spectroscopic investigation showed evidences of intermolecular bonding between epoxy and chitosan particles. The absorption peak was found to increase with chitosan loading.

\section{Conflict of Interests}

The authors declare that there is no conflict of interests regarding the publication of this paper.

\section{References}

[1] K. Dušek, J. Pleštil, F. Lednický, and S. Luňák, "Are cured epoxy resins inhomogeneous?” Polymer, vol. 19, no. 4, pp. 393-397, 1978.

[2] S. L. Selektor, M. Y. Semyachkin, and V. V. Arslanov, "A composite hardener for epoxy adhesives," Russian Journal of Applied Chemistry, vol. 76, no. 8, pp. 1331-1336, 2003.

[3] B. Qi, Q. X. Zhang, M. Bannister, and Y.-W. Mai, "Investigation of the mechanical properties of DGEBA-based epoxy resin with nanoclay additives," Composite Structures, vol. 75, no. 1-4, pp. 514-519, 2006.

[4] M. Tiitu, A. Talo, O. Forsén, and O. Ikkala, "Aminic epoxy resin hardeners as reactive solvents for conjugated polymers: polyaniline base/epoxy composites for anticorrosion coatings," Polymer, vol. 46, no. 18, pp. 6855-6861, 2005.

[5] Z. Zhang and N. Yu, "Studies on isotactic poly(phenyl glycidyl ether)-modified epoxy resins. II. Toughening of epoxy resins,"
Journal of Applied Polymer Science, vol. 84, no. 6, pp. 1223-1232, 2002.

[6] M. Ochi, T. Morishita, S. Kokufu, and M. Harada, "Network chain orientation in the toughening process of the elastomer modified mesogenic epoxy resin," Polymer, vol. 42, no. 24, pp. 9687-9695, 2001.

[7] V. Nigam, D. K. Setua, and G. N. Mathur, "Failure analysis of rubber toughened epoxy resin," Journal of Applied Polymer Science, vol. 87, no. 5, pp. 861-868, 2002.

[8] V. L. Pereira Soares, V. D. Ramos, G. W. M. Rangel, and R. S. V. Nascimento, "Hydroxy-terminated polybutadiene toughened epoxy resin: chemical modification, microstructure, and impact strength," Advances in Polymer Technology, vol. 21, no. 1, pp. 2532,2002 .

[9] Y.-L. Liu, Y.-J. Chen, and W.-L. Wei, "Novel thermosetting resins based on 4-(N-maleimidophenyl)glycidylether: I. Preparation and characterization of monomer and cured resins," Polymer, vol. 44, no. 21, pp. 6465-6473, 2003.

[10] M. R. Zhang, A Review of the Epoxy Resin Toughening, Department of Chemical Engineering and Materials Science, Syracuse University, Syracuse, NY, USA, 2003.

[11] H. Cai, J. Wang, X. Wang, and R. Xu, "Isocyanate-terminated polyethers toughened epoxy resin: chemical modification, thermal properties, and mechanical strength," Journal Wuhan University of Technology, Materials Science Edition, vol. 22, no. 2, pp. 256-260, 2007.

[12] R. D. Brooker, A. J. Kinloch, and A. C. Taylor, "The morphology and fracture properties of thermoplastic-toughened epoxy polymers," Journal of Adhesion, vol. 86, no. 7, pp. 726-741, 2010.

[13] R. A. Pearson and A. F. Yee, "Toughening mechanisms in thermoplastic-modified epoxies: 1. Modification using poly(phenylene oxide)," Polymer, vol. 34, no. 17, pp. 3658-3670, 1993.

[14] I. Aranaz, R. Harris, and A. Heras, "Chitosan amphiphilic derivatives. Chemistry and applications," Current Organic Chemistry, vol. 14, no. 3, pp. 308-330, 2010.

[15] S. Ritzenthaler, E. Girard-Reydet, and J. P. Pascault, "Influence of epoxy hardener on miscibility of blends of poly(methyl methacrylate) and epoxy networks," Polymer, vol. 41, no. 16, pp. 6375-6386, 2000.

[16] K. Mimura, H. Ito, and H. Fujioka, "Improvement of thermal and mechanical properties by control of morphologies in PESmodified epoxy resins," Polymer, vol. 41, no. 12, pp. 4451-4459, 2000.

[17] S. A. Shokralla and N. S. Al-Muaikel, "Thermal properties of epoxy (DGEBA)/phenolic resin (NOVOLAC) blends," The Arabian Journal for Science and Engineering, vol. 35, no. 1B, pp. 7-14, 2010.

[18] S.-J. Park, F.-L. Jin, and J.-R. Lee, "Thermal and mechanical properties of tetrafunctional epoxy resin toughened with epoxidized soybean oil," Materials Science and Engineering A, vol. 374, no. 1-2, pp. 109-114, 2004.

[19] E. Mahapoka, P. Arirachakaran, A. Watthanaphanit, R. Rujiravanit, and S. Poolthong, "Chitosan whiskers from shrimp shells incorporated into dimethacrylate based dental resin sealant," Dental Materials Journal, vol. 31, no. 2, pp. 273-279, 2012.

[20] M. G. González, J. G. Cabanelas, and J. Baselga, "Applications of FTIR on epoxy resins-identification, monitoring the curing process, phase separation and water uptake," in Infrared Spectroscopy-Materials Science, Engineering and Technology, T. Theophanides, Ed., pp. 261-284, InTech, 2012. 
[21] C. Radhakumary, P. D. Nair, S. Mathew, and C. P. R. Nair, "Biopolymer composite of chitosan and methyl methacrylate for medical applications," Trends in Biomaterials and Artificial Organs, vol. 18, no. 2, pp. 117-124, 2005.

[22] M. Rutnakornpituk, P. Ngamdee, and P. Phinyocheep, "Preparation and properties of polydimethylsiloxane-modified chitosan," Carbohydrate Polymers, vol. 63, no. 2, pp. 229-237, 2006. 

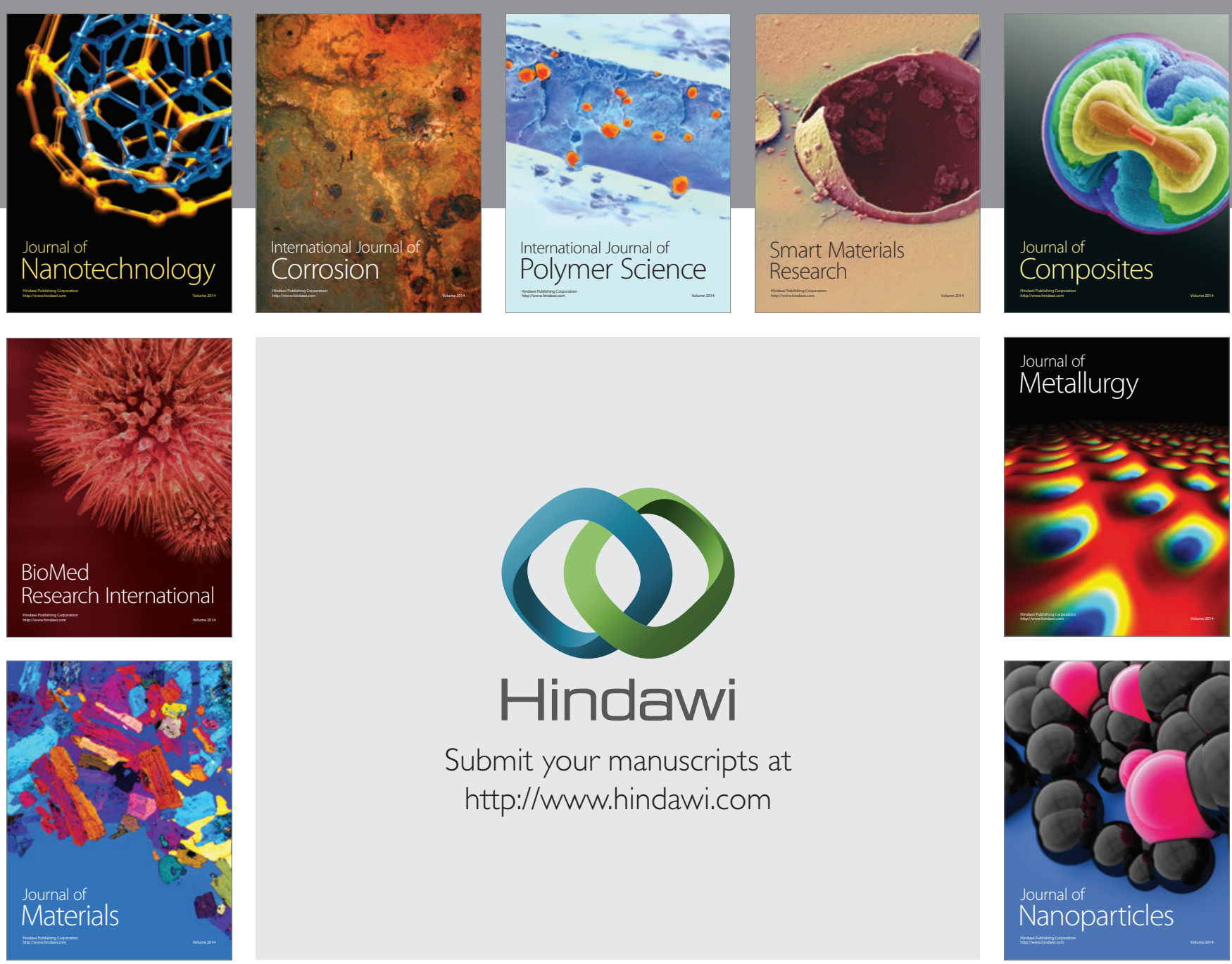

Submit your manuscripts at http://www.hindawi.com
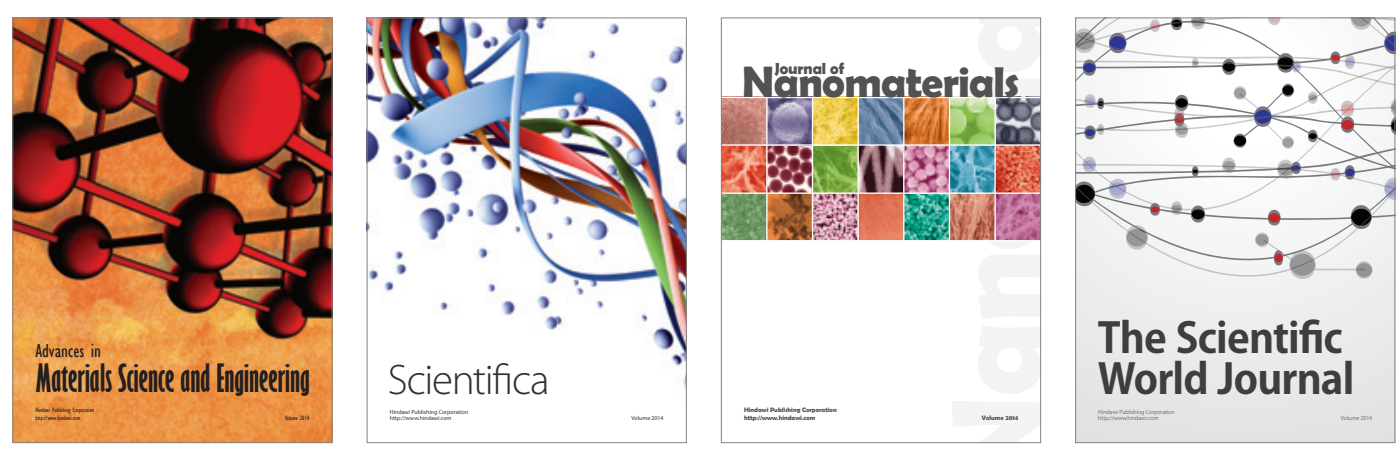

\section{The Scientific World Journal}
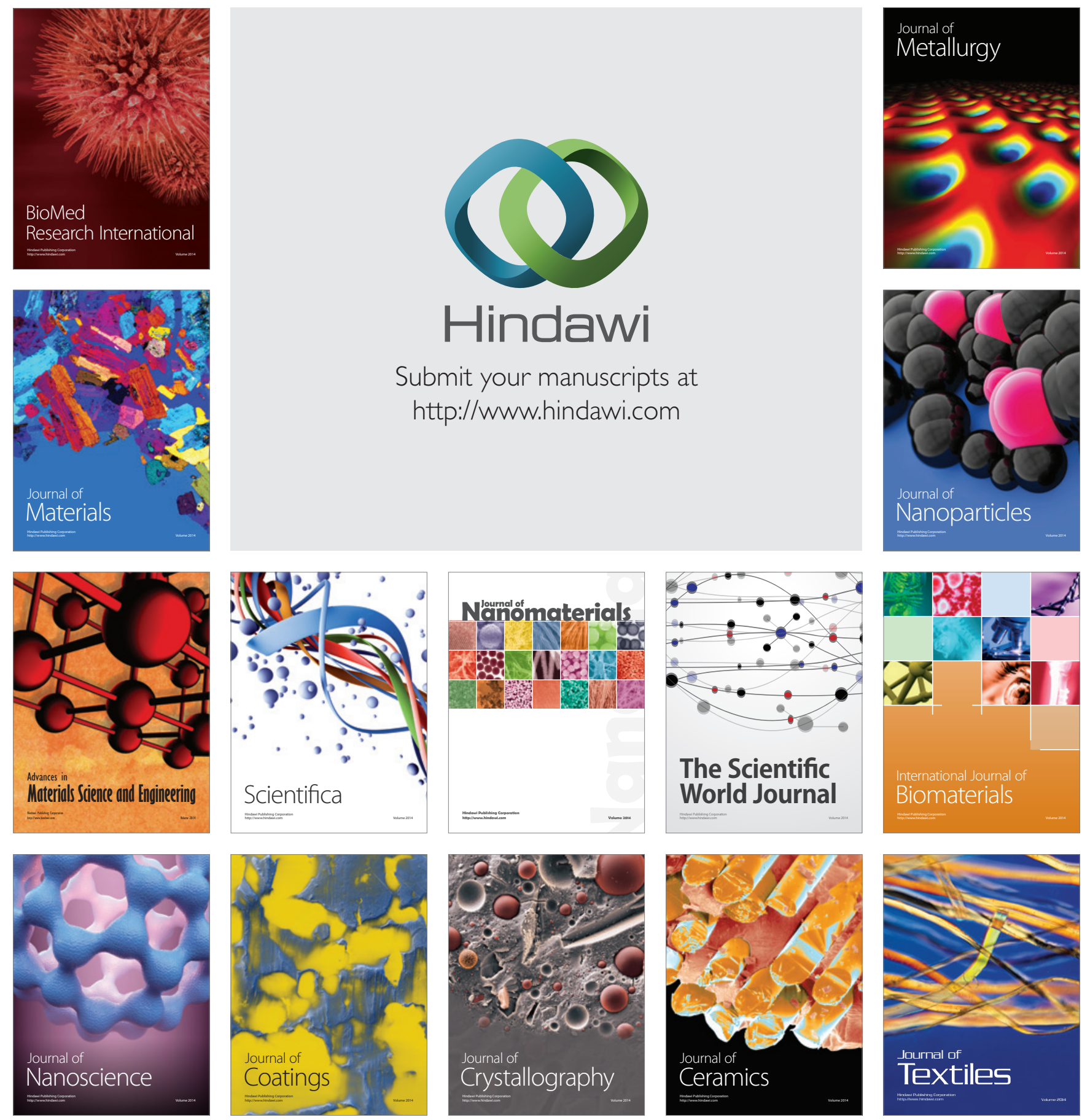